\title{
«Ich möchte die Ärztinnen und Ärzte an der Front erreichen»
}

\author{
Das «Swiss Medical Weekly» (SMW), eine englischsprachige Fachzeitschrift für me- \\ dizinische Forschung mit Open-access-Zugang im Internet (www.smw.ch), gehört in \\ seiner Impact-factor-Kategorie zur Spitzengruppe in Kontinentaleuropa. Im Juli dieses \\ Jahres wurde beim SMW eine grundlegende konzeptuelle Neuausrichtung realisiert, \\ über die Chefredaktor André Perruchoud* im folgenden Interview Auskunft gibt.
}

Interview:

Céline Fäh, Bruno Kesseli

* Prof. Dr. med. André Perruchoud, Facharzt für Innere Medizin und Pneumologie FMH, war bis zu seiner Pensionierung im Jahr 2007 Chefarzt der Medizinischen Klinik A des Universitätsspitals Basel und Dekan der Medizinischen Fakultät der Universität Basel. Er stiess 1997 zum Redaktions team des Swiss Medical Weekly und leitet die Zeitschrift seit 2008 als Chefredaktor.

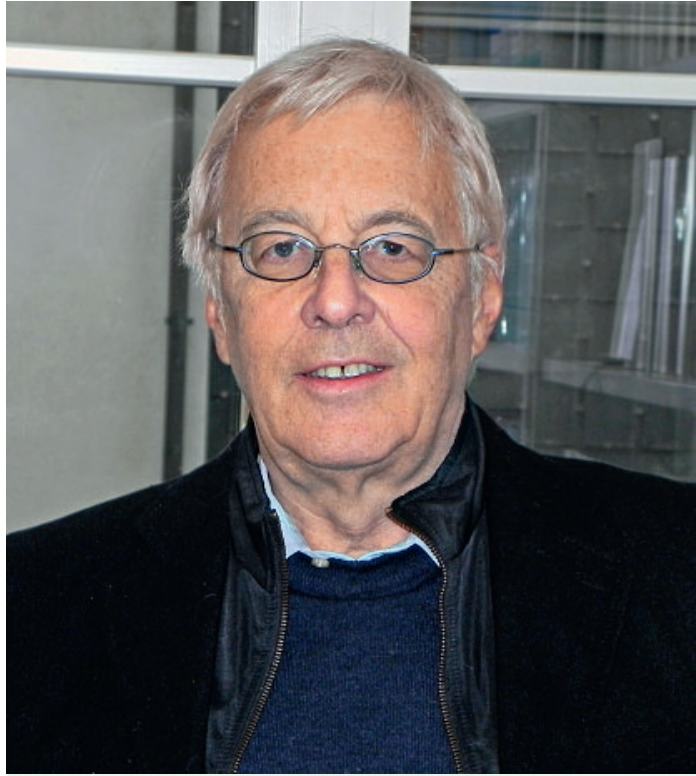

Möchte mit dem Swiss Medical Weekly eine optimale Plattform für den schweizerischen akademischen Nachwuchs anbieten: Chefredaktor André Perruchoud.

Seit Juli dieses Jahres ist beim Swiss Medical Weekly einiges anders als früher - man könnte fast von einem «Systemwechsel» sprechen. Was wurde verändert und aus welchem Grund?

\section{«Das SMW folgt dem}

\section{allgemeinen Trend, gehört aber} zu den Pionieren»

André Perruchoud: Wir haben keinen Systemwechsel vorgenommen, sondern die Entwicklung des Swiss
Medical Weekly vorangetrieben mit dem unveränderten Ziel, eine optimale Plattform für den schweizerischen akademischen Nachwuchs aufzubauen. Einerseits werden die Originalarbeiten laufend und sehr rasch elektronisch publiziert, was die Forscher sehr freut, anderseits halten wir an einer Printversion fest. Sie erscheint monatlich, enthält mehr Reviews und nur noch die erweiterten Zusammenfassungen der Originalarbeiten. Dies kommt dem allgemein orientierten Leser sicher sehr entgegen.

Folgt das SMW mit der Aufwertung der Online-Ausgabe einem Trend, der für medizinische Fachzeitschriften allgemein gilt, oder gehört die Zeitschrift in dieser Hinsicht zu den Pionieren?

«Open access» ist ein grosses Anliegen der Forschungsgemeinschaft, der Fakultäten und Schweizerischen Akademie der Medizinischen Wissenschaften. Viele, auch prominente Zeitschriften haben sich sehr positiv dazu geäussert - nur ganz wenige haben es in voller Konsequenz realisiert. Das SMW folgt also dem allgemeinen Trend, gehört aber zu den Pionieren. SMW wälzt übrigens die Druckkosten bisher nicht auf die Forscher ab. Das ist wirklich die grosse Ausnahme!

Welches Zielpublikum sprechen Sie mit dem SMW an? Gibt es Erkenntnisse darüber, ob Sie es auch erreichen?

Als Chefredaktor einer in der Schweiz publizierten Zeitschrift möchte ich die allgemein wissenschaftlich interessierten Leser erreichen. Ich meine damit nicht nur die Forscher, sondern die breite Ärzteschaft, welche ein langes akademisches Studium und einen universitären Abschluss hinter sich hat. Sie merken, wohin ich will? Ich möchte die Ärztinnen und Ärzte an der Front erreichen. Ich bin überzeugt, dass auch sie grosses Interesse an Forschung, am Stand der Kenntnisse und der Innovationen in der Medizin haben. Diesen Beweis muss ich selbstverständlich noch erbringen. 


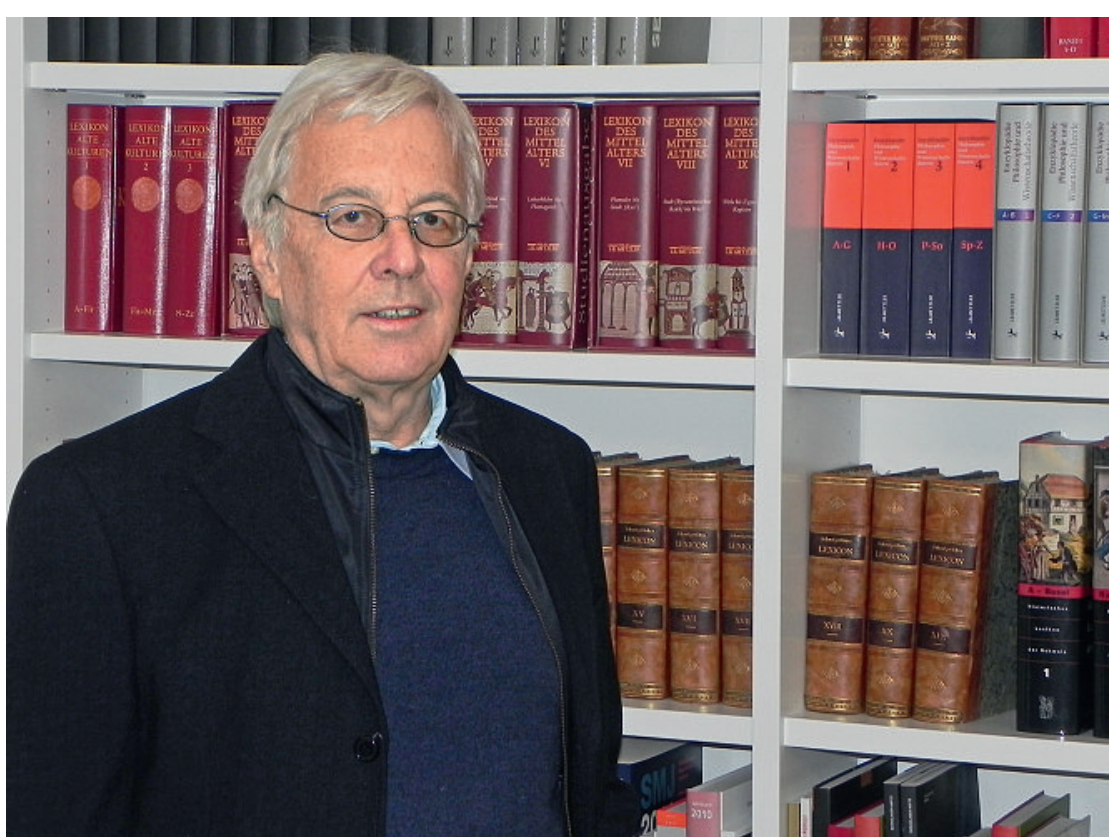

Versprechen Sie sich von den vorgenommenen Veränderungen auch die Erschliessung neuer Leserkreise?

Ja, ich bin überzeugt davon, dass unsere Kolleginnen und Kollegen sich für Übersichtsartikel (Medical intelligence), die Meinung von hoch anerkannten klinischen Experten (Current opinion), die technischen Neuerungen (Medtech), die unseren medizinischen
Der IF stellt eine wichtige Messgrösse dar. Nicht so sehr für den einzelnen Forscher, aber für den Stellenwert einer Zeitschrift. Er muss auch in diesem Bereich mit Vorsicht interpretiert werden, da sehr verschiedene Faktoren eine Rolle spielen, die auch, aber nicht nur mit der Qualität der eingereichten Manuskripte zu tun haben. Trotzdem arbeiten wir energisch daran, einen Impact Factor von 3,0 zu erreichen - dann wären wir in einer vernünftigen Liga! Zurzeit steigt der IF unserer Zeitung so, dass wir dieses Ziel in 4 Jahren erreichen könnten. Ein gewisses Niveau ist auch in dieser Hinsicht wichtig: on ne prête qu'aux riches!

Neben dem Impact Factor wird bei wissenschaftlichen Zeitschriften oft auch die Ablehnungsquote verglichen. Können Sie uns dazu etwas sagen?

Wir haben eine hohe Ablehnungsquote, sie liegt aktuell bei $79 \%$. Viele Arbeiten werden direkt nach einer Erstbeurteilung abgelehnt. Von den Arbeiten, die aufgrund dieser ersten Beurteilung der Redaktoren in den Reviewprozess eingeschleust werden, schaffen es die Hälfte zur Publikation.

Auf der Website des SMW ist festgehalten: «Die Qualität der Beiträge im «Swiss Medical Weekly> wird durch konsequentes internationales Peer reviewing garantiert.» Was muss man sich darunter vorstellen?

Jede Arbeit wird durch mindestens zwei externe Reviewer beurteilt. Wir legen Wert darauf, dass mindestens einer dieser beiden Reviewer aus dem Ausland kommt.

\section{«Trotzdem arbeiten wir energisch daran, einen Impact Factor von 3,0 zu erreichen - dann wären wir in einer vernünftigen Liga»}

Alltag bald prägen werden, und für den konzisen Überblick über aktuelle Forschungsresultate begeistern lassen. Es ist naiv zu behaupten, dass diese selbständig denkenden und arbeitenden Fachleute sich nur für «leichte Kost» interessieren.

Für medizinische Journals spielt der «Impact Factor» (IF) eine wichtige Rolle. Daran gemessen, zählt das SMW mit einem IF von knapp 1,7 zu den führenden Fachzeitschriften Kontinentaleuropas in der Kategorie «Medicine, general and internal». Wie bewerten Sie die Bedeutung des Impact Factors allgemein und für das SMW im Besonderen?
Die Manuskripte beinhalten sehr oft statistische Überlegungen. Deshalb werden diese Arbeiten noch von einem professionellen Statistiker begutachtet.

Haben Sie weitere Ziele, die Sie mit dem SMW in den kommenden Jahren erreichen wollen?

Bestimmt - lassen Sie mich zwei nennen: Als erstes möchte ich hier nochmals den Impact Factor von 3,0 anführen. Andererseits arbeite ich darauf hin, dass das SMW zwar nicht auf dem Nachttisch, aber vielleicht auf dem Bürotisch der praktisch tätigen Ärztin und des praktisch tätigen Arztes zu finden ist. 